\section{Allergologische Innovationen}

\section{Allergologie lernt von Transplantationsmedizin}

\author{
Immunmodulatorische Therapien spielen bei der Behandlung des \\ atopischen Ekzems eine immer größere Rolle. Besondere Bedeutung \\ kommt dabei den Makroliden wie Tacrolimus (FK 506) zu, wie \\ auf der 41. Tagung der Deutschen Dermatologischen Gesellschaft in \\ Berlin zu hören war.
}

D as aus der Transplantationsmedizin altbekannte Immunsuppressivum Cyclosporin A (CyA) ist in Deutschland auch zur Behandlung schwerer Fälle von atopischem Ekzem zugelassen. Bis zu 80\% der Patienten sprechen auf die Behandlung an. Der Juckreiz lässt nach wenigen Tagen nach, das Ekzem verschwindet oft binnen weniger Wochen. Doch wenige Wochen nach Absetzen von CyA erleben die meisten ein erneutes Aufflammen der Erkrankung, bemängelte Dr. Kristian Reich in seinem Vortrag zur Therapie des atopischen Ekzems. Der Dermatologe von der Universität Göttingen empfiehlt niedrig dosiertes CyA als Langzeitbehandlung oder Kurzzeit-Intervalltherapie, womit er Remissionen bis zu mehreren Monaten nach Absetzen erreicht habe. Aufgrund der Toxizität einer systemischen CyA-Behandlung sollte sie jedoch weitgehend Therapie-resistenten Patienten vorbehalten bleiben.

Einen wesentlichen Fortschritt in der Therapie des atopischen Ekzems markiert nach Ansicht von Reich die Einführung der topisch wirksamen Immunmodulatoren wie Tacrolimus.

\section{Von der Nierentransplantation zum atopischen Ekzem}

1984 entdeckte eine japanische Pharmafirma, dass der Bodenpilz Streptomyces tsukubaensis eine immunsuppressive Substanz bildet, und isolierte daraus Tacrolimus. Der seit mehreren Jahren bei Organtransplantationen eingesetzte Wirkstoff dringt in Entzündungszellen ein und bindet mit zyto- plasmatischen Proteinen an das Enzym Calcineurin. Dieser Komplex unterbricht die Reaktionskaskade in TZellen, die nach Antigen-Stimulierung zur Bildung von Zytokinen wie IL-12 führt. Eine Wirkung wurde auch an basophilen und eosinophilen Granulozyten beobachtet, an Keratinozyten und Antigen-präsentierenden Zellen.

In Japan und den USA ist Tacrolimus als 0,1\%ige Creme zur Behandlung des atopischen Ekzems auf dem Markt. Der Hersteller rechnet im nächsten Jahr
AAD-Kongress, Washington 2001

AAAAI-Kongress, New Orleans 2001 mit der europäischen Zulassung. Nach 28 Studien mit 5.000 Patienten sei laut Reich eine „klinische Besserung, die der Wirkung einer systemischen Cyclosporin-Therapie vergleichbar ist," zu erwarten. Studien zufolge tritt schon ab dem vierten Tag eine 40- bis 60\%ige Verbesserung des atopischen Ekzems ein. Nach drei Wochen wurde eine Besserung von $80 \%$ erreicht und auch nach sechs bis zwölf Monaten heilten mittelschwere bis schwere Ekzeme weiter ab.

Der Vorteil der topischen Immunmodulatoren liegt neben der guten Wirksamkeit vor allem im Ausbleiben schwerer Nebenwirkungen wie Hautatrophie oder Schädigung des Kollagenstoffwechsels. Lokale Irritationen nach Auftragen der Salbe wie Brennen und Juckreiz waren nach wenigen Tagen, parallel zum Abklingen der Symptome, verschwunden. Als Makrolid ist FK 506 relativ groß und kann die intakte Hautbarriere kaum überwinden. Systemische Nebenwirkungen sind daher unwahrscheinlich.

Symposium „Atopische Dermatitis im Kindes- und Erwachsenenalter", 41. DDGTagung, Berlin 2001

\title{
Neues Antihistaminikum auch in Deutschland zu haben
}

Auf dem Kongress der „American Academy of Dermatology“ (AAD), der Anfang März in Washington stattfand, präsentierte die Arbeitsgruppe um J. Ring (München) Ergebnisse einer Studie, die sie bei 190 Urtikaria-Patienten mit Desloratadin gegen Plazebo durchführte. Bereits die erste Desloratadin-Gabe erzielte eine JuckreizMinderung um 45\% - im Gegensatz zu 3,5\% nach Plazebo. Nach sechswöchiger Therapie waren fast $70 \%$ der Verum-Gruppe vs. $45 \%$ der Plazebo-Gruppe beschwerdefrei. Zudem konnten 35\% der Verum-Gruppe bereits in der ersten Nacht durchschlafen im Vergleich zu 15\% in der Plazebo-Gruppe. Nach 42 Tagen Behandlung mit Desloratadin hatten $80 \%$ der Patienten einen ungestörten Schlaf. In der Plazebo-Gruppe waren es nur 50\%. Diese Ergebnisse wurden, wie Ring auf dem AAAAI-Kongress in New Orleans ausführte, durch eine andere multizentrische, aber offene Phase-III-Studie bestätigt. An ihr beteiligten sich bisher 150 Patienten mit chronischer idiopathischer Urtikaria. Die Ergebnisse korrelierten sehr gut mit der auf dem AAD-Kongress vorgestellten Studie. So waren $51 \%$ der Patienten mit schwerer Urtikaria nach vier Wochen Behandlungszeit vollkommen beschwerdefrei. Bei den anderen Patienten war eine erhebliche Besserung der Symptome zu verzeichnen. Auch die Ergebnisse in der Verbesserung der Lebensqualität korrelierten gut mit der ersten Studie. Bereits nach einer Woche konnten ca. $50 \%$ der Patienten ohne Juckreiz nachts durchschlafen, nach vier Wochen waren es bereits über 70\%. Auch tagsüber nahm der Juckreiz beachtlich ab, nach einer Woche um etwa $50 \%$, nach vier Wochen um mehr als 55\%. Dabei unterschied sich die Phase-III-Studie in ihrem Nebenwirkungsspektrum nicht von den bekannten bei Plazebo beobachteten Effekten. 